\title{
Secure File Transfer Protocol
}

National Cancer Institute

\section{Source}

National Cancer Institute. Secure File Transfer Protocol. NCI Thesaurus. Code C100048.

A network protocol designed to move files securely between a computer and a host over a TCP network, such as the Internet. 